\title{
Um Modelo de Arquitetura para Gerenciar a Criação de Recursos Educacionais Abertos em Núcleos de EaD
}

\author{
Lanylldo Araujo dos Santos, Luís Carlos Costa Fonseca, \\ Reinaldo Silva de Jesus, Francisco da Conceição Silva
}

Programa de Pós-Graduação em Engenharia de Computação e Sistemas Universidade Estadual do Maranhão - UEMA

lanylldo@gmail.com, lccfonseca@gmail.com, fransconcfs@gmail.com, reinaldo.silvarrrb@gmail.com

\begin{abstract}
This article presents the partial results of a research that aim to propose an Educational Design tool to be used in the Distance Education Centers, as a computational resource to assist since the step of creating a courseware, through its packaging as a learning object (OA) using a metadata standard, to distribution in a repository of educational resources. To design the software they were considered $O A$ to create patterns in the literature and also a survey of information carried along with a multidisciplinary team that creates $O A$ in a University of Brazil.
\end{abstract}

Resumo. Neste artigo são apresentados os resultados parciais de uma pesquisa que têm como objetivo propor uma ferramenta de Design Educacional a ser utilizada em Núcleos de Educação a Distância, como um recurso computacional para auxiliar desde a etapa de criação de um material didático, passando pelo seu empacotamento como um objeto de aprendizagem (OA) utilizando um padrão de metadados, até a sua distribuição em um Repositório de recursos educacionais. Para a concepção do Software foram levados em consideração padrões de criação de OA existentes na literatura e ainda um levantamento de informações realizados junto a uma equipe multidisciplinar que cria OA em uma Universidade do Brasil.

\section{Introdução}

Em 2005 o Ministério da Educação (MEC) criou o Sistema Universidade Aberta do Brasil (UAB), em parceria com a Associação Nacional dos Dirigentes das Instituições Federais de Ensino Superior (ANDIFES) e Empresas Estatais. Isto ocorreu no âmbito do Fórum das Estatais pela Educação com foco nas Políticas e a Gestão da Educação Superior. Trata-se de uma política pública de articulação entre a Secretaria de Educação a Distância - SEED/MEC e a Diretoria de Educação a Distância - DED/CAPES (Coordenação de Aperfeiçoamento de Pessoal de Nível Superior) com vistas à expansão da educação superior, no âmbito do Plano de Desenvolvimento da Educação - PDE.

Desde então a política do Governo Federal para a o ensino à distância tem se respaldado no repasse de recursos às instituições públicas de ensino superior para a ampliação do acervo bibliográfico dos polos de apoio presencial, fomentar a criação de novos cursos e apoiar a formação de professores.

Atualmente, são mais de 100 instituições integrantes do Sistema UAB, entre Universidades Federais, Universidades Estaduais e Institutos Federais de Educação, 


\section{CBIE-LACLO 2015}

Anais dos Workshops do IV Congresso Brasileiro de Informática na Educação (CBIE 2015)

Ciência e Tecnologia (IFETs). De 2007 a julho de 2009, foram aprovados e instalados 557 polos de apoio presencial com 187.154 vagas criadas. A UAB, ademais, em agosto de 2009, selecionou mais 163 novos polos, no âmbito do Plano de Ações Articuladas, para equacionar a demanda e a oferta de formação de professores na rede pública da educação básica, ampliando a rede para um total de 720 polos (ABED, 2015).

Os números apresentados demonstram um massivo crescimento na oferta de cursos e vagas, e consequentemente no número de disciplinas. Nesse cenário surge um grande desafio, que é o de gerenciar a produção de conteúdo para estas disciplinas, cumprindo prazos e mantendo a qualidade dos conteúdos produzidos, assim como a adequação à modalidade à distância.

Para administrar tantas disciplinas e consequentemente atores, pessoas envolvidas no processo de produção de conteúdo, as instituições integrantes do Sistema UAB criaram os chamados Centros de Educação à Distância $(\mathrm{CEaD})$, sendo que esta nomenclatura pode variar em cada instituição, algumas chamam de Núcleos de Educação à Distância (NEaD) ou Núcleo de Tecnologias Educacionais (NTE).

Atualmente, os CEaDs cuidam de todas as fases de produção de conteúdos digitais para educação à Distância $(\mathrm{EaD})$, que vão desde a editoração de Cadernos de Estudos, passando pela gravação de vídeoaulas, podcasts, screencasts, transmissão de videoconferências até a catalogação armazenamento destes conteúdos em Repositórios de Objetos de Aprendizagem (ROA). A tarefa de controle de tantas atividades de produção se tornou um desafio de gestão, e é ai onde entram as metodologias de produção de conteúdo (abordadas em momento oportuno neste artigo) e as ferramentas de automação desta gestão.

Este trabalho propõe um modelo de arquitetura computacional que pretende automatizar o controle do processo de produção de conteúdos digitais para $\mathrm{EaD}$ até o empacotamento dele na forma de um Objeto de Aprendizagem (OA). O artigo está organizado em cinco seções, sendo que a primeira é esta introdução; uma segunda trata do referencial teórico. Na terceira apresentamos a metodologia utilizada para a pesquisa. $\mathrm{Na}$ sequência, a quarta seção os resultados da pesquisa. E por último, são tecidas as considerações finais e perspectivas de trabalhos futuros.

\section{Referencial Teórico}

Esta seção tem o papel de apresentar o aporte teórico utilizado como fundamento deste trabalho. Os conceitos apresentados a seguir nortearam as atividades dessa pesquisa, bem como influenciaram a concepção do Software.

\subsection{Produção de Objetos de Aprendizagem como REA}

Antes de iniciar a discussão desta seção é importante definir o conceito de Recurso Educacional Aberto (REA). A Unesco entende REA como:

\footnotetext{
"materiais de ensino, aprendizado e pesquisa em qualquer suporte ou mídia, que estão sob domínio público, ou que estão licenciados de maneira aberta, permitindo que sejam utilizados ou adaptados por terceiros. $\mathrm{O}$ uso de formatos técnicos abertos facilita o acesso e o reuso potencial dos recursos publicados digitalmente. Recursos Educacionais Abertos podem incluir cursos completos, partes de cursos, módulos, livros didáticos, artigos de pesquisa, vídeos, testes, software e qualquer outra ferramenta, material ou técnica que possa apoiar o acesso ao conhecimento" (Butcher, p.42, 2015).
}

Nesse sentido, uma das funcionalidades chave para esta ferramenta é que ela dê suporte ao reuso de conteúdo. Para tanto é importante que os recursos desenvolvidos na plataforma sejam abertos, e isto implica na preocupação com licenças de uso. Vejamos abaixo dois cenários onde esta preocupação é relevante: 


\section{CBIE-LACLO 2015}

Anais dos Workshops do IV Congresso Brasileiro de Informática na Educação (CBIE 2015)

1. Imagine que um Professor A pode fazer a segunda oferta de uma mesma disciplina ministrada anteriormente pelo Professor B. Na nova versão, o Professor A gostaria de aproveitar alguns materiais, modificar o plano de ensino e o formato de algumas aulas, além de inserir conteúdos de autoria própria.

2. Em uma segunda situação, existe uma disciplina que compartilha assuntos com outra e o professor que vai ministrar a nova cadeira gostaria de aproveitar apenas alguns materiais, mas o maior volume é de sua própria autoria.

Em ambos os casos, se não houver um licenciamento adequado dos conteúdos, um professor que deseje reaproveitá-los pode ter problemas legais sérios. Para o caso da plataforma que está sendo proposta neste trabalho, considera-se inclusive o reuso do planejamento do curso ou partes dele.

A legislação brasileira é muito protetora no que se refere aos direitos autorais, segundo a lei 9610/98 que trata dos direitos autorais no Brasil (Brasil, 1998) o que não é expressamente aberto se torna automaticamente fechado. Portanto, em uma plataforma onde o reuso é característica chave, a preocupação com a clareza das licenças é primordial. Vejamos o que trata o artigo 49 da lei 9610/98:

Art. 49. Os direitos de autor poderão ser total ou parcialmente transferidos a terceiros, por ele ou por seus sucessores, a título universal ou singular, pessoalmente ou por meio de representantes com poderes especiais, por meio de licenciamento, concessão, cessão ou por outros meios admitidos em Direito, obedecidas as seguintes limitações:

I - a transmissão total compreende todos os direitos de autor, salvo os de natureza moral e os expressamente excluídos por lei;

II - somente se admitirá transmissão total e definitiva dos direitos mediante estipulação contratual escrita;

III - na hipótese de não haver estipulação contratual escrita, o prazo máximo será de cinco anos. (Brasil, 2015).

Para (MORAIS et al., 2013), o movimento REA vai além de recursos é um processo de engajamento com os recursos didáticos, focando em: usar e adaptar o que foi criado por outros para o seu próprio uso; compartilhar o que você cria sozinho ou em conjunto com outros professores/alunos; compartilhar novamente o material que você adaptou, de forma que outros usuários possam ser beneficiados.

Para entender melhor como funciona a produção dos REA, vale a pena pensar em todo um "ciclo de vida" para o recurso educacional. "Na perspectiva de um professor, esse ciclo começa com uma tarefa que faz parte do cotidiano: o desejo ou a necessidade de aprender ou ensinar algo (MORAIS et al., 2013)"; em seguida pode-se tentar encontrar algum recurso que fundamente ou complemente o conteúdo investigado; depois parte-se para as tarefas de criação, adaptação às necessidades pedagógicas e aplicação; ficando por último o compartilhamento da produção, formando assim um ciclo retroalimentado.

Nesse sentido, o ciclo de vida dos REA é perfeitamente aderente aos processos de produção de OAs e por isso se torna um conceito relevante para a fundamentação deste trabalho. Convém ressaltar que a licença aberta, onde um autor concede os direitos autorais para terceiros é o que diferencia um REA de um OA, em razão disso, o processo de confecção de um REA é igual ao de um OA.

\subsection{Modelos de Processos de Construção de OA}

Na literatura, o conceito de padrão de processo para a construção de OA é explicado por (PESSOA e BENITTI, 2007) como uma estratégia utilizada para fazer com que os serviços ou rotinas sejam impessoais, ou seja, independente de quem executará os trabalhos, serão realizadas as mesmas atividades, avaliados os mesmos parâmetros e produzidos os mesmos artefatos. Esses padrões têm como objetivo garantir a qualidade 


\section{CBIE-LACLO 2015}

Anais dos Workshops do IV Congresso Brasileiro de Informática na Educação (CBIE 2015)

na criação de objetos de aprendizagem, por meio da padronização de rotinas e funções desenvolvidas pelos profissionais comprometidos com a confecção de OA.

Ao encontro do que menciona (PESSOA e BENITTI, 2007) sobre processos de criação de OA, o padrão de Processos Unificados Racional (RUP, 2002) define quatro elementos essenciais para a criação de um padrão. $O$ primeiro, a Etapa/Fase corresponde ao tempo entre dois marcos primários do projeto, durante o qual um conjunto bem definido de objetivos é atendido, artefatos são concluídos e decisões são tomadas. O segundo é referente ao Artefato e pode ser entendido como uma informação que é usada ou produzida por um processo de desenvolvimento, em outras palavras, um artefato é o produto gerado por uma Atividade, como um documento de descrição de um OA ou um software. O próximo conceito elencado pelo autor trata-se do Papel que é uma definição do comportamento e das responsabilidades de um colaborador ou de uma equipe de colaboradores; Por fim, o último elemento Atividade: uma ação de trabalho designada a um ou mais colaboradores.

O segundo modelo de construção de OA foi criado pela Rede Interativa Virtual de Educação (RIVED), e por isso se chama RIVED, faz parte de um programa da Secretaria de Educação a Distância vinculado ao Ministério da Educação do Brasil. O RIVED tem como objetivo a produção de matérias pedagógicos digitais, e o seu processo produtivo possui seis fazes que estão elencadas abaixo (Rived, 2015).

- Fase 1: criação de um documento, por parte da equipe de educadores e técnicos, chamado de General Design (GD) com a descrição das atividades e objetivos.

- Fase 2: O documento GD é submetido à equipe para obter críticas e feedbacks.

- Fase 3: os professores (as) de cada disciplina revisam o conteúdo e revisam as indicações emitidas pela Fase 2 e criam especificações para cada OA.

- Fase 4: criação do objeto.

- Fase 5: os professores (as) de cada disciplina desenvolvem os manuais ou guias de uso para cada OA.

- Fase 6: os objetos são organizados e publicados na web.

Por fim, o último modelo de processo estudado é chamado de Sophia. Este foi criado junto ao repositório de conteúdos educacionais digitais chamados de Ambiente Sophia, conforme (VILLELA, 2004), os três estágios existentes no Sophia para o desenvolvimento de OA são a etapa de Projeto, representada pela criação de um Projeto do material didático contendo informações de quais conceitos e tecnologias o mesmo irá abordar, nesta fase, também é detalhado a função de cada membro da equipe e o tempo para criação do recurso. A seguir o OA entra em Desenvolvimento, onde o mesmo é criado levando em consideração o tempo e as atribuições de papeis definidas na fase anterior, após a criação o novo objeto entra no estado de Distribuição, nesta etapa o objeto é empacotado em um padrão de metadados, depositado em um repositório e ao fim do processo, são realizadas avaliações sobre a qualidade e desempenho do OA com alunos (as) e professores (as) que utilizam o OA.

Após descrever os dois modelos, mesmo que brevemente, é fácil perceber que entre os padrões RIVED e Sophia existem ações comuns como: a priori, planejar a criação do material e registrar essas atividades no plano didático contendo os papéis dos colaboradores e objetivos didáticos do $\mathrm{OA}$; a posteriori, a criação do recurso educacional seguindo as recomendações estabelecidas no plano didático, e ao fim anexar ao novo OA um padrão de metadados, distribuí-lo em um repositório ou em um 


\section{CBIE-LACLO 2015}

Anais dos Workshops do IV Congresso Brasileiro de Informática na Educação (CBIE 2015)

Ambiente Virtual de Aprendizagem (AVA), e realizar avaliações para conhecer os impactos causados pelo mesmo.

\section{Metodologia}

As atividades desenvolvidas neste trabalho foram baseadas no conceito de um estudo de caso e por isso, conforme explica (Gil, 2002) a pesquisa foi divida em quatro fases essenciais, são elas: a) delimitação da unidade-caso; b) coleta de dados; c) seleção, análise e interpretação dos dados; d) elaboração do relatório.

A primeira fase consiste em delimitar a unidade ou área em que se constitui o estudo, sendo assim, nesta pesquisa o cenário escolhido foi uma Universidade pública do Brasil que possui vínculo com a UAB e com o Programa Nacional de acesso ao Ensino Técnico e Emprego (PRONATEC). Esta Universidade através do seu NEAD está presente como polo presencial de $\mathrm{EaD}$ em 35 municípios brasileiros, com cerca de 10.000 (dez mil) discentes matriculados em 24 cursos gratuitos à distância, sendo 12 cursos de nível técnico financiados pelo PRONATEC; 6 especializações em nível de pós-graduação financiados pela UAB; 4 cursos de graduação também mantidos pela UAB; e 2 cursos mantidos na plataforma de Cursos Abertos (MOOC - Massive Open Online Course) (NEAD, 2015).

Por conta desses cursos, a Universidade possuiu um acervo de materiais didáticos que cresce a cada dia, um exemplo disso são os 54 cadernos de estudos criados para os cursos técnicos entre o período de 2012 a 2014, que são distribuídos através de mídia impressa para todos os alunos (as), bem como tem sua versão digital depositada no repositório do PRONATEC mantido pelo MEC (NEAD, 2015).

Em média, são enviados para cada discente 25 exemplares, considerando que cada curso tem aproximadamente 25 disciplinas. Para as disciplinas que ainda não tem material didático criado são utilizados obras distribuídas por outras instituições de ensino que estão no repositório do MEC. Além destes, também são produzidos outros OAs como: vídeoaula, fascículos, avaliações, animações utilizadas nos vídeos e imagens. Dessa forma, portanto, com as características citadas justifica-se a escolha dessa Universidade como a unidade-caso onde esta pesquisa se desenvolve.

A segunda fase deste trabalho corresponde à coleta de dados que representa, conforme explica (SOMMERVILLE, 2007), o levantamento de requisitos para criação de uma ferramenta consiste em criar um documento contendo as descrições e regras de negócios sobre o ambiente para o qual o software será utilizado. Essa etapa aconteceu durante um período de três meses, através de observações, anotações, cópias de arquivos e conversas com colaboradores da instituição, onde foi possível compreender as atividades existentes no processo de confecção de um recurso educacional realizado por uma equipe multidisciplinar.

Durante a visita ao NEAD, foi possível perceber dois aspectos importantes, sendo o primeiro, a variedade de materiais didáticos produzidos pela Universidade, e o outro, se refere ao critério de tempo (dias) utilizado pela mesma para selecionar os tipos de recursos que serão criados por um professor (a) em uma disciplina. Por exemplo, quando o processo de criação dispõe de 75 dias é possível criar vídeoaulas com animações, registrar o livro criado com ISBN (Padrão Internacional de Número do Livro), e utilizar todas as atividades disponíveis para o ambiente de aprendizagem. Vale ressaltar que, essa relação entre tempo (dias) e recurso a ser criado, foi uma medida adotada pela instituição a fim de garantir a qualidade nos novos objetos criados.

Após a coleta de informações, teve início a seleção de dados para a criação dos casos de uso e diagramas utilizando a Linguagem de Modelagem Unificada (UML), onde se modelou as principais ações e processos envolvidos na criação de um OA. 


\section{CBIE-LACLO 2015}

Anais dos Workshops do IV Congresso Brasileiro de Informática na Educação (CBIE 2015)

Dessa forma, foi possível criar um documento com uma breve descrição das rotinas de produção de um material didático. A partir desse relatório foi possível ter noção de como gerenciar e fazer com que as várias ações de criação, edição e revisão aconteçam em prazos determinados e sincronizados, fazendo com que cada membro da equipe desempenhe seu papel sem sobrecarga. Convém citar, que essa equipe é formada por mais de 50 pessoas com profissionais de diferentes áreas de conhecimento e especialistas em tecnologias.

Portanto, podemos postular que os relatos de experiências citados nos parágrafos anteriores demonstram as principais ações realizadas nessa pesquisa. Sendo assim, percebe-se que todas as atividades acima tinham como objetivo levantar informações sobre uma problemática que envolve as principais dificuldades encontradas em um NEAD durante a confecção de OAs. Logo após o estudo de caso ter fim, surgiu à necessidade de pensar em um software que atendesse as dificuldades encontradas durante o estudo realizado no NEAD.

\section{Resultados Alcançados}

Nesta seção será exposta a concepção de um recurso computacional que padroniza e gerencia a criação de recursos educacionais. Para isto, faremos uso da UML na representação do software que foi concebido a partir dos modelos de criação de OA, bem como das regras de negócio e procedimentos colhidos junto ao NEAD da Universidade, como foi exemplificado na Metodologia deste.

\subsection{A Ferramenta de Design Educacional}

O Software de Design Educacional é uma ferramenta de gestão do processo de criação e armazenamento de OA. O sistema tem como papel suprir a necessidade de núcleos de EAD em organizar e padronizar o fluxo de rotinas necessárias para a criação de recursos tecnológicos educacionais. E ainda, publica os novos matérias em um ROA.

Nesse sentido, a ferramenta constitui-se um recurso computacional responsável por administrar os diferentes papéis de em uma equipe multidisciplinar durante o desenvolvimento de um novo recurso. Esse processo é composto basicamente por atividades técnicas e pedagógicas, sendo que as atividades técnicas correspondem à gravação e edição de áudio, vídeo, imagem e texto; enquanto as atividades pedagógicas equivalem à criação delimitação dos fins didáticos do $\mathrm{OA}$, bem como, $\mathrm{o}$ acompanhamento de todo o processo de criação pelos educadores.

Por conta das suas características, o software proposto nesta pesquisa é categorizado como um Sistema de Gerenciamento de Conteúdos de Aprendizagem (LCMS), pois, o mesmo, é um ambiente no qual educadores e especialistas em mídias podem criar, armazenar, reutilizar, controlar e publicar o material criado em um repositório (Litto, 2009).

Nessa perspectiva, Greenberg (2002), explica que um bom LCMS apresenta uma infraestrutura com componentes que possibilitam o planejamento, criação e distribuição de recursos tecnológicos educacionais nos principais formatos de vídeo, áudio, imagem, texto e hipertexto, bem como suporta múltiplos sistemas de autoria se integrando facilmente com o LCMS.

Após, essa descrição e categorização sobre o software de Design Educacional, a seguir será apresentado às principais funcionalidades, casos de uso e diagrama da arquitetura do LCMS proposto neste trabalho.

\subsection{Arquitetura}

O protótipo do LCMS, como já mencionado anteriormente, foi modelado com o papel de suprir a necessidade dos núcleos $\mathrm{EaD}$ em padronizar o fluxo de rotinas na criação de materiais didáticos, convém explicar agora que esta padronização, segundo (PESSOA e 


\section{CBIE-LACLO 2015}

Anais dos Workshops do IV Congresso Brasileiro de Informática na Educação (CBIE 2015)

BENITTI, 2007) está relacionada com a necessidade de possuir uma equipe multidisciplinar formada por diferentes tipos de profissionais que possam responder de forma coerente, pelo caráter tanto pedagógico, quanto técnico, buscando com isso a padronização dos processos e uma melhor qualidade dos OAs.

Desse modo, a arquitetura do software foi modelada com funções que possuem a capacidade de gerenciar uma equipe com perfis heterogêneos, nos moldes delineados pelo autor. A Figura 1 apresenta a arquitetura do software, bem como, os seus três subsistemas, os atores identificados até o momento e a base de dados.

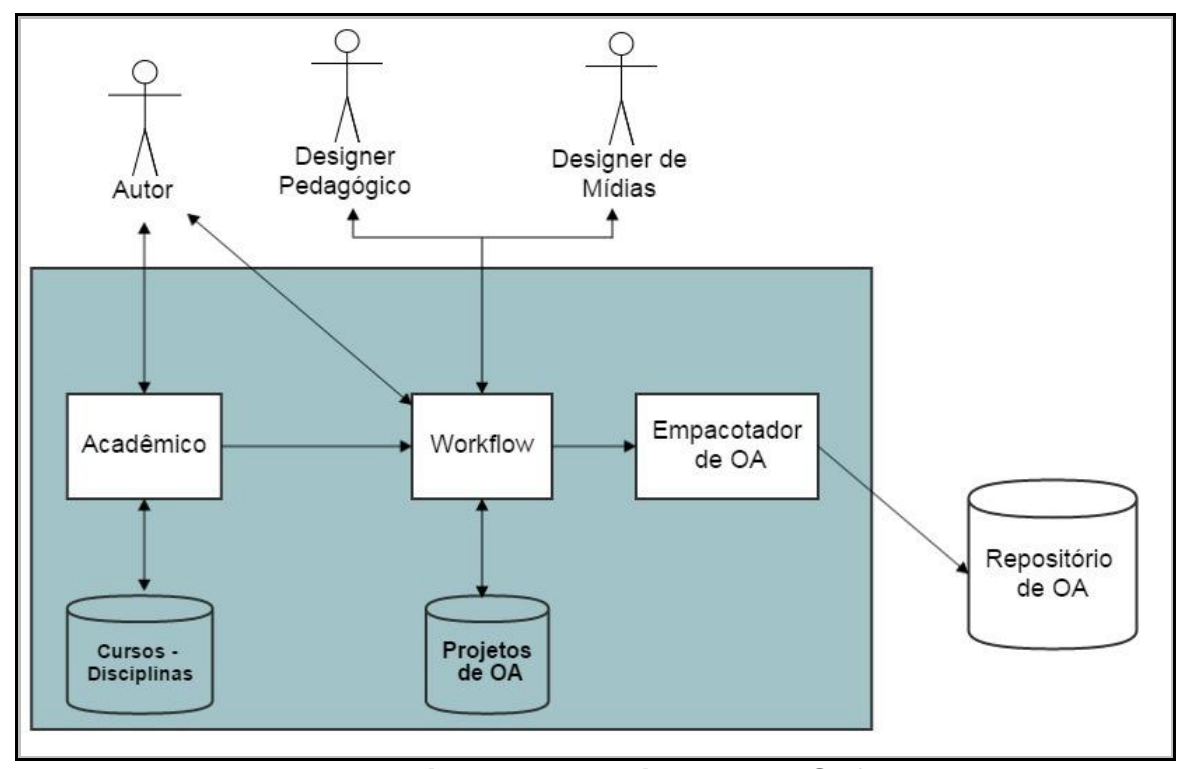

Figura 1: Arquitetura do Software

Conforme é apresentado na Figura 1, a arquitetura computacional é formada por três subsistemas, são eles:

- Acadêmico - gerencia as informações relacionadas ao controle de disciplinas dos cursos. Em outras palavras, este módulo do software tem o intuito de organizar todos os recursos criados em disciplinas e as disciplinas em um curso, sendo assim, quando um curso ou partes dele for ofertado novamente todo o seu conteúdo pode ser reaproveitado. É importante ressaltar que a solução encontrada para esta funcionalidade tornou-se possível através da modelagem do banco de dados do ambiente, pois quando um conteúdo é reaproveitado ele é armazenado em um novo espaço no banco de dados possibilitando que o novo curso possa sofre alterações no seu conteúdo sem prejuízos para o curso antigo. Desse modo, é viável ter o controle de versões de cada curso com os seus respectivos OAs.

- Workflow - subsistema de gestão das rotinas de criação de um objeto de aprendizagem. Por ser o principal recurso do Software, o Workflow é utilizado por todos os colaboradores e foi pensado levando em consideração as três ações, comuns, existentes nos modelos RIVED e Sophia, de Planejar, Criar seguindo o planejamento e Distribuir o novo objeto em um ROA. Dessa forma, o papel do Workflow é organizar as atribuições de cada colaborador no desenvolvimento dos recursos apresentados na Tabela 1, e armazenar cada novo material na base de dados, assim como as alterações realizadas no material. Empacotador - tem o intuito de empacotar o material didático na forma de um OA, ou seja, anexar junto ao recurso educacional, os seus metadados, seguindo 


\section{CBIE-LACLO 2015}

Anais dos Workshops do IV Congresso Brasileiro de Informática na Educação (CBIE 2015)

um padrão. Os metadados são utilizado a fim de resolver problemas de interoperabilidade entre repositórios de recursos educacionais, no Brasil existe um Padrão de Metadados de Objetos de Aprendizagem mas conhecido pela sigla Obaa (VICARI et al. 2010) que será a referencia, a princípio, para empacotar os OAs.

Conforme foi apresentado acima, na arquitetura do software é importante registrar que os módulos Acadêmico e Workflow já possuí uma versão funcional tanto de software quando de banco dedados. Para esta versão esta sendo foi utiliza o Spring MVC framework que utiliza a linguagem de programação Java e o banco de dados Mysql. Ainda em relação ao empacotador, durante o desenrolar das atividades desta pesquisa, surgiu à necessidade de criar um repositório para armazenar recursos didáticos e a partir disso, vincular esse repositório com o LCMS, a fim de que esses recursos tenham como ser empacotados como um OA e depositados em uma ROA automaticamente.

Para suprir essa necessidade, foi desenvolvido um sistema responsável por armazenar e distribuir os OAs na Universidade. Esse repositório foi implementado a partir do software Dspace que é de código fonte aberto e foi criado pelo Instituto de Tecnologia do Massachusetts (MIT), o sistema gerencia os OAs nos principais formatos de mídia e gerencia o acesso a esses recursos educacionais. A Figura 2 ilustra a primeira versão do ROA que esta em fase de teste na Universidade.

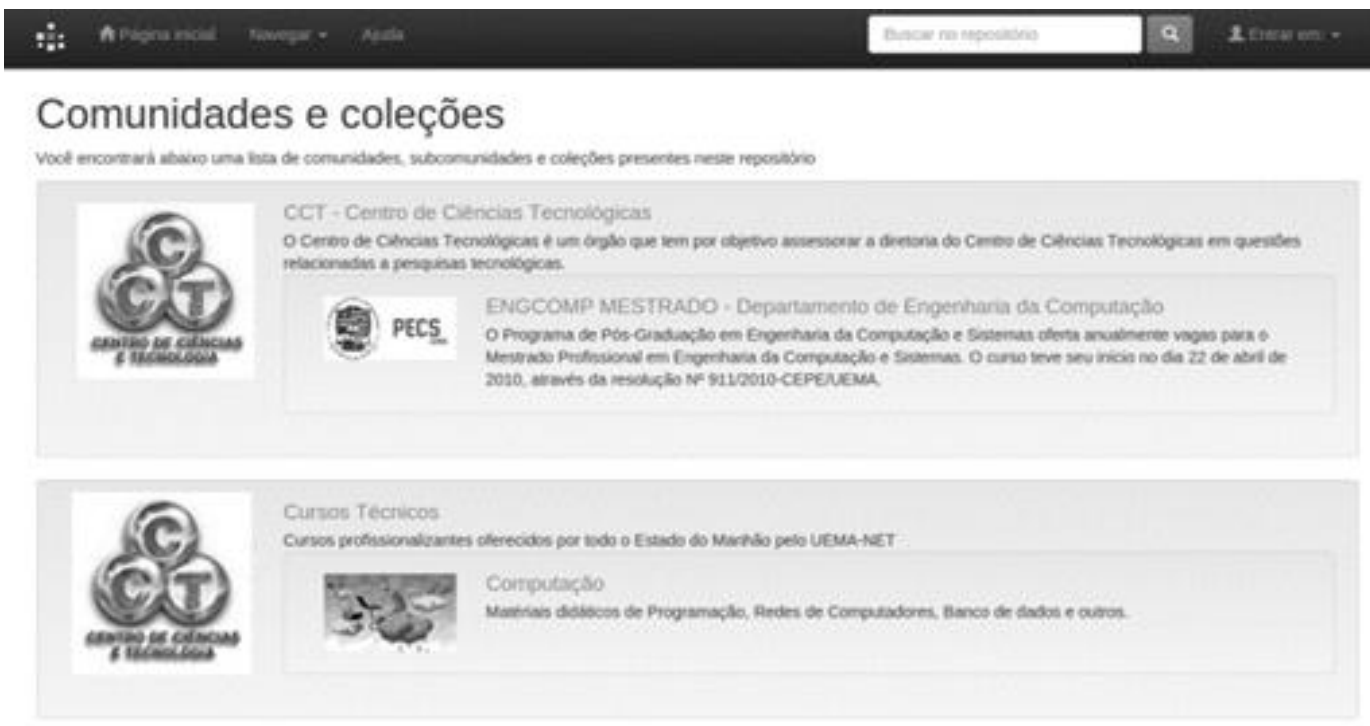

Figura 2: Repositório da Universidade

Com a apresentação dos módulos do LCMS é interessante, nessa altura do texto, detalhar os casos de uso já identificados para a ferramenta, dentre eles, foi possível definir três tipos de colaboradores/atores que fazem parte do processo de criação do OA. O primeiro colaborador identificado no sistema é chamado de Autor e sua função está relacionada à criação de todo o conteúdo, como por exemplo, quais conceitos científicos e culturais serão abordados em um curso e consequentemente pelos objetos. A Figura 3 mostra o caso de uso do Autor. 


\section{CBIE-LACLO 2015}

Anais dos Workshops do IV Congresso Brasileiro de Informática na Educação (CBIE 2015)

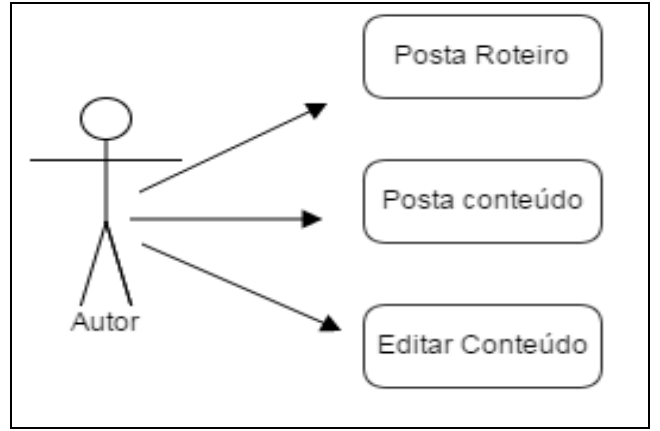

Figura 3 : Caso de uso Autor

O autor, como pode ver visto na Figura 3, possuí três atribuições, sendo uma, a postagem do roteiro contendo as descrições pedagógicas e os conceitos abordados no OA, as outras duas representam a postagem de conteúdo e sua edição, este conteúdo, podem ser os capítulos de um livro, questões de uma avaliação, tópicos de um fórum e outros. Convém exemplificar que essa edição corresponde às alterações enviadas por outro ator chamado de Designer de Mídias (DM), o qual uma de suas funções é avaliar e/ou enviar correções de todo o conteúdo postado pelo Autor.

O segundo ator identificado é chamado de Designer Pedagógico (DP) e suas atuações na ferramenta estão incluídas em acompanhar e supervisionar os procedimentos realizados pelos colaboradores Autor e Designer de Mídias, com o objetivo de criar condições para que os prazos e metas estabelecido na construção do OA sejam cumpridos. O caso de uso da Figura 5 apresenta as funções do DP.

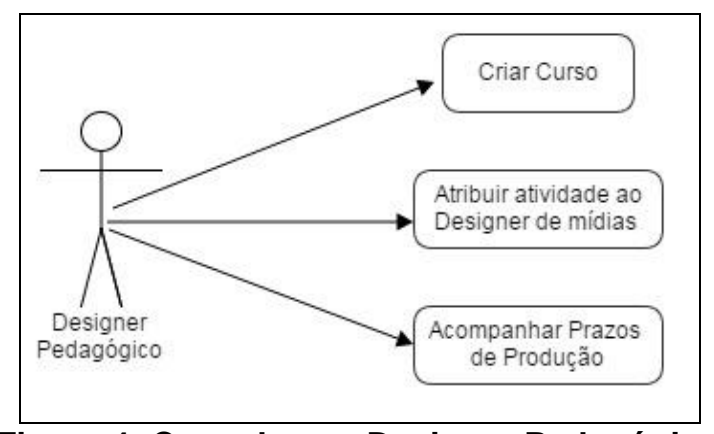

Figura 4: Caso de uso Designer Pedagógico

Em relação a Figura 4, podemos postular que as atividades do DP, em síntese, são criar um novo curso com suas respectivas disciplinas, atribuir função ao DM, ou seja, delimitar quais os colaboradores que irão participar da criação de um novo material. Vale frisar que o DM corresponde aos profissionais especialistas em uma área, como é o caso do técnico em videoconferência, revisor de mídias textuais e editor de vídeo. Após delimitar os papéis de todos os envolvido na criação, o DP acompanha todo o processo de desenvolvimento supervisionando as atividades com o intuito de criar um novo recurso de qualidade e dentro do tempo previsto.

\section{Considerações Finais}

Neste artigo foram apresentados os resultados parciais de uma pesquisa que têm como objetivo propor uma ferramenta, a ser utilizada em Núcleos de Educação a Distância, como um recurso computacional para auxiliar na criação de OA.

Foi apresentada uma arquitetura, o repositório de materiais didático já criado, bem como um número de diagramas e modelos que refletem o estágio atual de desenvolvimento da ferramenta. Como perspectiva futura pretende-se fazer experimentos em ambiente de produção de um NEAD de uma Universidade pública. 


\section{CBIE-LACLO 2015}

Anais dos Workshops do IV Congresso Brasileiro de Informática na Educação (CBIE 2015)

Para tanto, será escolhida uma quantidade de disciplinas onde utilizaremos a ferramenta para gerenciar a produção de conteúdo.

\section{Considerações Finais}

Esta trabalho foi financiado pela Fundação de Amparo à Pesquisa e ao Desenvolvimento Científico e Tecnológico do Maranhão - FAPEMA.

\section{REFERÊNCIAS}

ABED. Associação Brasileira de Educação a Distância. Censo EaD.br: Relatório Analítico da Aprendizagem a Distância no Brasil 2013.São Paulo, 2014.

BRASIL. Lei $\mathrm{N}^{\mathrm{o}}$ 9.610, de 19 de fevereiro de 1998. Disponível em:< http://www.planalto.gov.br/19610.htm> Acessado em: 15 de novembro de 2014.

BUTCHER, N. (Author), KANWAR, A. (Ed.), e UVALIC-TRUMBIC, S. (Ed.). 2011). A Basic Guide to Open Educational Resources (OER). Vancouver, Canada: Commonwealth of Learning, and Paris, France: UNESCO.

LITTO, Educação a Distância: O estado da arte. São Paulo: Pearson, 2009

GIL, A. C. Como Elaborar Projetos de Pesquisa. São Paulo: Atlas, 2002.

GREENBERG, L. LMS and LCMS: Whats the Difference? Learning Circuits, American Society for Training and Development, ASTD. 2002.

MORAIS, E.; RIBEIRO, A.; AMIEL.T.; Recursos Educacionais Abertos: Um caderno para professores. São Paulo: Educação Aberta, 2013.

MUSTARO, P. N., Silveira, I. F., Omar, N. e Stump, S. M. D. (2007), "Structure of Storyboard for Interactive Learning Objects Development", Learning Objects: and instructional design, Koohang, A. and Harman, K., Santa Rosa, Informing Science Press, p.253-279.

NEAD. "Sobre o NEAD da Universidade Estadual do Maranhão". Disponível em:< http://www.uema.br > Acessado em: 15 de março 2015.

(NEAD, 2015).

PESSOA, M.C.; BENITTI, F.B.V.; Proposta de um Processo para Produção de Objetos de Aprendizagem. Hífen, Uruguaiana, II semestre 2008. Disponivel em: $<$ http://revistaseletronicas.pucrs.br/3483>. Acesso em: 1 de maio de 2015

VICARI, R.M et al. Proposta Brasileira de Metadados para Objetos de Aprendizagem Baseados em Agentes (Obaa). Disponível em: <http://seer.ufrgs.br/article /15257/9015> Acessado em: 30 de abril de 2015.

RUP. Rational Unification Process: release notes (version 2002.05.00). [S.1]: Rational Software Corporation, 2002.

RIVED (2015), “Conheça o RIVED”, Disponível em: $<$ http://www.rived.mec.gov.br/conheca_rived.php>. Acessado em: 15 abril 2014.

SOMMERVILLE, L. Engenharia de Software. São Paulo: Pearson Education, 2007.

VILLELA, K. (2004) "Definição e Construção de Ambientes de Desenvolvimento Software Orientados à Organização", Tese de D.Sc., COPPE/UFRJ, Rio de Janeiro, RJ, Brasil, maio. Disponível em <http://www.cos.ufrj.br/taba > Acessando em 15 de abril de 2015.

WAZLAWICK, R. S. Metodologia de Pesquisa para Ciência da Computação. Rio de Janeiro: Elsevier, 2009. 